Z Rheumatol 2020 $79: 837-838$

https://doi.org/10.1007/s00393-020-00902-3

Angenommen: 18. September 2020

(c) Springer Medizin Verlag GmbH, ein Teil von Springer Nature 2020

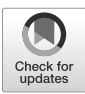

\author{
H.-I. Huppertz ${ }^{1}$ U. Müller-Ladner ${ }^{2,3}$ \\ 'Bremen, Deutschland \\ ${ }^{2}$ Lehrstuhl für Innere Medizin, Schwerpunkt Rheumatologie, Justus-Liebig-Universität Gießen, Gießen, \\ Deutschland \\ ${ }^{3}$ Abteilung für Rheumatologie und Klinische Immunologie, Justus-Liebig-Universität Gießen, Campus \\ Kerckhoff, Bad Nauheim, Deutschland
}

\title{
Aktive Impfungen
}

\section{Eine der wesentlichen Maßnahmen zum Erhalt der Gesundheit unserer Patienten mit rheumatischen Erkrankungen}

nachhaltigen Wandel erfahren. Sicher geglaubte Errungenschaften wie Freizügigkeit der persönlichen Entfaltung in Europa oder die weltweite Reisetätigkeit im Rahmen der Globalisierung sind nur noch eingeschränkt möglich. Außerhalb der eigentlichen Infektion sind die Folgen zudem für viele Menschen existenziell bedrohlich. Die von den Staaten ergriffenen Maßnahmen konnten die Pandemie in ihrer Wucht zwar einigermaßen begrenzen, und insbesondere Deutschland hat bisher einen im internationalen Vergleich recht glücklichen Verlauf erlebt. Viele Bevölkerungsanteile wie chronisch Kranke, ältere Mitbürger, Behinderte, aber auch Kinder haben besonders darunter gelitten (siehe z.B. [1]).

\section{\) Bedeutung von Impfungen in der Pandemie}

Weltweit hofft man daher auf eine baldige Entwicklung von Impfungen gegen den Erreger [2], wobei auch hier schon zu beobachten ist, dass sich einzelne Staaten in Position bringen, um möglichst früh möglichst viele der Impfstoffe der eigenen Bevölkerung zur Verfügung stellen zu können. Positiv ist, dass sich aufgrund der drängenden Notwendigkeit viele Forscher und Impfstoffhersteller sehr intensiv Gedanken machen, an welcher Stelle ein SARS-CoV-2-Impfstoff angreifen könnte, was sicherlich die Impfstoffentwicklung auch für kommende infektiolo- gische Probleme nachhaltig beeinflusst. Obwohl die Zulassungen noch ausstehen, werden die ersten Impfstoffe bereits produziert.

Das aktuelle Impfwesen mit den vorhandenen vielfältigen Impfstoffen hat in Deutschland ein sehr hohes Niveau erreicht. Im vorliegenden Band der Zeitschrift für Rheumatologie soll dargestellt werden, wie dieses Wissen am besten für unsere Patienten mit rheumatischen Erkrankungen nutzbar gemacht werden kann. Dabei soll auch ein allgemeines Verständnis für die Grundlagen des Impfwesens geboten werden.

Hiermit verbunden ist auch das Problem der Impfgegner, welches sich besonders in den alten Bundesländern hartnäckig, zum Teil in malignem Ausmaß hält und die Gesundheit aller Vernünftigen nachhaltig bedroht. Nachvollziehbare Argumente werden hierbei nicht vorgebracht, zumeist soll nur ihre eigene, auch ökonomisch motivierte Agenda unterstützt werden.

Dass approbierte Kolleginnen und Kollegen dabei die wissenschaftlich begründete Medizin verlassen und die in der Berufsordnung niedergelegten Pflichten nicht adäquat achten, ist umso tragischer für die betroffenen Patienten zu werten. Je besser wir, insbesondere als immunologisch ausgebildete Rheumatologen, informiert sind, desto leichter und überzeugender können wir hier auf allen Kommunikationsebenen qualifiziert entgegentreten. den. Weltweit hat das tägliche Leben einen dramatischen und wahrscheinlich 
Thomas Harder vom Robert KochInstitut beschreibt im ersten Beitrag, wie das Impfwesen in Deutschland organisiert ist. Gesetzlich geregelt, spricht die Ständige Impfkommission (STIKO) Empfehlungen zur Durchführung von Impfungen aus. Sie tut dies auf einer jeweils aktuell erarbeiteten, außerordentlich breiten wissenschaftlichen Grundlage nach einem klar beschriebenen und transparenten Vorgehen. Zudem werden alle möglichen Maßnahmen ergriffen, um die Unabhängigkeit der STIKO und ihrer Mitglieder zu dokumentieren und $\mathrm{zu}$ erhalten. Das Besondere der Empfehlung der STIKO ist, dass diese von den obersten Gesundheitsbehörden der Länder übernommen werden in den Katalog der von den gesetzlichen Krankenkassen $\mathrm{zu}$ bezahlenden Impfungen, wenn der gemeinsame Bundesausschuss einer entsprechenden Empfehlung nicht innerhalb von 3 Monaten widersprochen hat.

Patienten mit rheumatischen Erkrankungen werden nicht selten mit Immunsuppressiva behandelt. Daniel Schreiner und Fred Zepp aus der Universitätskinderklinik Mainz beschreiben die allgemeinen Prinzipien des Impfens unter Immunsuppression. Totimpfstoffe sind im Allgemeinen ohne signifikantes Risiko zu applizieren, die Schutzwirkung kann in Stärke und Dauer jedoch nicht ganz der Effektivität bei Gesunden entsprechen. Lebendimpfungen sind im Allgemeinen kontraindiziert, einzelne Impfungen können unter bestimmten Bedingungen durchgeführt werden. Die ärztliche Kunst besteht darin, auch diesen Patienten einen möglichst optimalen Schutz durch Impfungen zu gewähren.

Wie so häufig liegt beim individuellen Patienten das Problem im Detail. Christian Kneitz und Ulf Müller-Ladner beschreiben in ihrem Beitrag die spezifischen Möglichkeiten des Impfens bei Patienten mit rheumatischen Erkrankungen. Wenn irgend möglich, sollten alle Impfungen erfolgt sein, bevor die immunsuppressive Therapie begonnen wird. Aber auch unter den meisten der in der Rheumatologie eingesetzten Therapien sind Impfungen möglich, die Gabe muss aber individualisiert werden und ist abhängig von der jeweiligen Impfung, vom Ausmaß der Immunsuppression, der behandelten Grundkrankheit und dem Ansprechen auf diese Therapie. Zudem beeinflussen individuelle Faktoren das Vorgehen. Alle Patienten mit rheumatischen Erkrankungen sollen daher die saisonale Influenzaimpfung im Herbst erhalten sowie einen adäquaten Schutz gegen Pneumokokken und angesichts des häufigeren Auftretens von Herpes zoster unter Januskinasehemmern auch die Totimpfung gegen das Varizella-Zoster-Virus.

Obwohl die interkontinentale Reisetätigkeit aktuell weitgehend zum Erliegen gekommen ist, spielen Reiseimpfungen bei rheumatischen Erkrankungen weiterhin eine große Rolle. In einem exzellenten Artikel beschreiben Tatjana Welzel, Andreas Wörner und Ulrich Heininger vom Kinderspital beider Basel, welche Impfungen für welchen Patienten zu empfehlen sind.

Die Zeit der Pandemie hat für alle noch einmal klargemacht, wie sehr Gesundheit auch vom solidarischen Verhalten aller abhängt. Unsere Patienten mit rheumatischen Erkrankungen profitieren von einem solidarischen Verhalten, d.h. von der Durchführung von Impfungen zum frühestmöglichen Zeitpunkt bei allen, für die diese Impfungen empfohlen sind. $\mathrm{Ne}$ ben der Bedeutung der Herdenprotektion können wir unseren Patienten auch helfen, indem wir ihnen die vorhandenen Impfungen anbieten und mit unserem Wissen und unserer Überzeugungskraft dazu raten, diese durchführen zu lassen. Entsprechend wünschen wir Ihnen, liebe Leserin und lieber Leser, Spaß und Erkenntnisgewinn beim Lesen des vorliegenden Heftes.

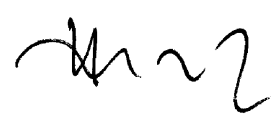

\section{H.-I. Huppertz}

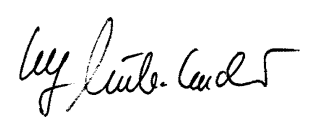

U. Müller-Ladner

\section{Korrespondenzadresse}

Prof. Dr. med. H.-I. Huppertz

Schwachhauser Heerstr. 163a, 28211 Bremen, Deutschland

hihuppertz@hotmail.de

Interessenkonflikt. H.-I. Huppertz und U. MüllerLadner geben an, dass kein Interessenkonflikt besteht.

\section{Literatur}

1. Deutsche Akademie für Kinder- und Jugendmedizin Stellungnahme der Deutschen Akademie für Kinder- und Jugendmedizin e. V. zu weiteren Einschränkungen der Lebensbedingungen von Kindern und Jugendlichen in der Pandemie mit dem neuen Coronavirus (SARS-CoV-2). www.dakj. de.Zugegriffen:20.Apr. 2020

2. Slaoui M, Hepburn M (2020) Developing safe and effective covid vaccines-operation warp speed's strategy and approach. N Engl J Med. https://doi. org/10.1056/NEJMp2027405 